\title{
Los Centros de Cultura Popular e Indígena Yaqui desde la perspectiva de sus Promotores Culturales
}

Dr. Cristian Salvador Islas Miranda Lic. Brian Neftalí Osuna Verdugo Instituto Tecnológico de Sonora

Resumen: La presente investigación tiene como objetivo caracterizar los procesos de enseñanza y de gestión utilizados por los promotores culturales para el fortalecimiento del patrimonio cultural de seis centros de cultura de la comunidad yaqui de Sonora: Loma de Guamúchil, Tórim, Pótam, Belém (Pitahaya), Ráhum y Huírivis. Para ello se desarrolló un diseño de investigación con un enfoque cualitativo y alcance descriptivo financiado por el Programa de Fomento y Apoyo a los Proyectos de Investigación (PROFAPI) del Instituto Tecnológico de Sonora. Se entrevistó a los promotores culturales que habitan en la misma comunidad en junio del 2019. El instrumento estuvo compuesto por 19 preguntas dividido en cuatro categorías: función del centro de cultura, enseñanza aprendizaje, financiamiento y estrategias para la salvaguardia del patrimonio cultural. Se encontró que en cada pueblo es el único espacio de esparcimiento y de formación artística. Es empleado para impartir talleres de arte tradicional con el objetivo de fortalecer la preservación del patrimonio cultural de la comunidad yaqui: clases de bordado, lengua cahíta, danza de venado, clases de violín, guitarra popular, dibujo, creación de cabezas de venado con papel, entre otras. No cuentan con una fuente económica permanente para la compra de materiales y generación de nuevos proyectos por lo que dependen, principalmente, del Programa de Apoyo a las Culturas Municipales y Comunitarias (PACMyC). También utilizan dinero propio para adquisición de consumibles. De manera esporádica reciben donaciones en especie. Los centros de cultura tienen una función clave en la generación de lazos entre los músicos y danzantes tradicionales de los ochos pueblos yaquis, ya que prestan las instalaciones para realizar los encuentros de gastronomía, música popular, pascolas y venados, desde su creación en los años ochenta, coordinados por el Centro de Culturas Populares e Indígenas de Cajeme y la Dirección General de Culturas Populares, Indígenas y Urbanas.

Palabras clave: Patrimonio cultural, Etnia yaqui, Centros de cultura

\section{Introducción}

Los centros de cultura promueven los valores culturales entre los miembros de una comunidad donde se localizan; se estructura en torno a espacios amplios donde tienen lugar diferentes manifestaciones culturales que enriquecen y animan la vida cultural de la población local (DeCarli, 2012); por lo regular estos espacios cuentan con una biblioteca, área para talleres y presentaciones culturales y también se acondicionó una sala donde se llevan a cabo exposiciones temporales; suelen ser abiertos al público y tener un carácter multifuncional con el objetivo de incidir en la promoción y la cooperación cultural (Vives, 2009).

En este marco, el presente estudio surgió por el interés de atender el vacío referencial relacionada con los centros de cul- 
tura de la región yaqui y su trabajo con la comunidad yaqui, que a pesar de los cambios administrativos a nivel federal, se mantienen activos desde 1984 y son los únicos espacios que se encargan de la formación artística cultural en cada pueblo. Por lo tanto, se emprende el estudio desde un enfoque descriptivo para identificar las opciones culturales recreativas que se ofrecen en los centros, la manera en que son difundidas y cuáles son los objetivos culturales, artísticos, sociales y educativos a alcanzar, así como su compromiso con el patrimonio cultural local en la actualidad desde la visión de los mismos promotores.

\section{Desarrollo}

En el estado de Sonora existen los Centros de Cultura Popular de Sonora, que están ubicados en la mayoría de las regiones pertenecientes a los pueblos originarios como Yaqui, Mayo, Seri, Guarijío y Cucapá. Estos nichos son utilizados principalmente para la capacitación de los mismos habitantes a través de talleres multidisciplinarios, investigación y difusión de sus culturas tradicionales. Como parte de su infraestructura cuentan con un área de exhibición etnográfica, de consulta bibliográfica y es utilizado para el desempeño de labores investigativas y administrativas propias (Castro, 2010). Sus antecedentes se remontan a 1978 con la creación de la Dirección General de Culturas Populares por iniciativa del reconocido catedrático y defensor de los derechos de los pueblos indígenas Dr. Rodolfo Stavenhagen (1932-2016) con el propósito de "impulsar una política cultural que procurara el fortalecimiento de los procesos y expresiones de las culturas populares como parte sustantiva de la cultura nacional" (ISC, s.f., parr. 6), mediante la promoción, estudio y conservación de las expresiones de la cultura popular e indígena, trabajando directamente con la comunidad. Fue hasta 1983 que se estableció la unidad regional de Sonora cuyas oficinas se ubicaron en la ciudad de Hermosillo.
Se capacitó como Promotores Culturales a oriundos de los mismos pueblos indígenas con cursos en la Ciudad de México, Hermosillo y en su misma comunidad, con lo que se formalizó su planta laboral como empleados de la Dirección General de Culturas Populares que inició como dependencia de la Secretaría de Educación Pública y posteriormente pasó a la Secretaría de Cultura (Castro, 2016).

De acuerdo con Tonatiuh Castro Silva, investigador de Culturas Populares, en la actualidad existen centros de cultura indígena en los siguientes municipios de Sonora:

Centro de Cultura Yaqui "Capitán Santiago Valencia Amarillas”.

Ubicación: Loma de Guamuchil, Cajeme

Inicio de actividades: 1983.

Centro de Cultura Yaqui "Capitán Manuel Molina León".

Ubicación: Tórim, Guaymas.

Inicio de actividades: 1983.

Centro de Cultura Yaqui "Capitán Juan María Santeamea".

Ubicación: Pótam, Guaymas.

Inicio de actividades: 1983.

Centro de Cultura Yaqui "Gral. Ignacio Mori Seamo".

Ubicación: Belém (Pitahaya), Guaymas.

Inicio de actividades: 1983.

Centro de Cultura Yaqui "José Bacasegua Seamo".

Ubicación: Rahúm, Guaymas.

Inicio de actividades: 1983.

Centro de Cultura Yaqui “Jesús Quintero

Valencia".

Ubicación: Huírivis, Guaymas.

Inicio de actividades: 1983.

Centro de Cultura Mayo "Blas Mazo". Ubicación: Júpare, Huatabampo.

Inicio de actividades: 1986. 
Centro de Cultura Mayo "Francisco Mumulmea Zazueta".

Ubicación: Buaysiacobe, Etchojoa

Inicio de actividades: 1988.

Centro de Cultura Seri "Coyote Iguana". Ubicación: Punta Chueca, Hermosillo. Inicio de actividades: 1985.

Centro de Cultura Seri "Chico Romero", Desemboque de los Seris.

Ubicación: Pitiquito.

Inicio de actividades: 1986.

Centro de Cultura Guarijío "José Zazueta Yoquivo".

Ubicación: Mesa Colorada, Álamos. Inicio de actividades: 1990.

Centro de Cultura Cucapá "Sipá y Komat".

Ubicación: Pozas de Arvizu, San Luis Río Colorado.

Inicio de actividades: 2011.

Centro de Culturas Populares e Indígenas de Cajeme.

Ubicación: Cajeme, Cd. Obregón.

Inicio de actividades: 1998.

Comunidad indígena yaqui

La mayor cantidad de población perteneciente a la comunidad indígena yaqui está ubicada en la parte sureste del estado de Sonora en los municipios de Guaymas, Bácum y Cajeme. La región está compuesta por un área serrana, la sierra de Bacatete, una costera y el valle, donde se localizan los campos agrícolas y el Distrito de Riego No. 18 (Castro, 2010). La etnia se halla distribuida principalmente en ocho pueblos tradicionales que son, de sur a norte: Loma de Guamúchil, Loma de Bácum, Tórim, Vícam, Pótam, Ráhum, Huirivis y Belem (INPI, 2018), originalmente llamados, Cócorit del Espíritu Santo, Santa Rosa de Bácum, San Ignacio de Tórim, La Natividad del Señor de Vícam, La Santísima Trinidad de Pótam, La Asunción de Ráhum, Santa Bár- bara de Huírivis y San Miguel de Belem. Desde una visión general, la vida cotidiana de la comunidad yaqui gira en torno a la religión católica y sus fiestas tradicionales, en una investigación relacionada con las ceremonias en los pueblos indígenas de Sonora, Muñoz (2018) considera que las agrupaciones cahítas (yaquis y mayos) y su arraigo cercano a los ríos Fuerte, Yaqui y Mayo, crearon una tradición festiva asociada a los ciclos agrícolas notablemente influidas por el advenimiento de la religión, "donde los rituales del calendario litúrgico católico se sobreponen o corresponden a las distintas etapas del ciclo agrícola y natural, tal como bendición de semillas, desmonte, preparación de la tierra, siembra, petición de lluvia, cultivo, cosecha y agradecimiento" (Muñoz, 2018, pág. 7).

Como parte de las fiestas tradicionales de la etnia yaqui, cada uno de los ocho pueblos tienen sus fiestas tradicionales que son ofrecidas a diferentes santos, pero dentro de las fiestas la más representativa es la cuaresma (Semana Santa) que se lleva a cabo en los meses de febrero abril. Según Muñoz (2018), la Cuaresma y Semana Santa se dividen en dos etapas: inicia el miércoles de ceniza y termina el domingo de ramos, es llamada pasión de fiestas. "En el miércoles de ceniza se utiliza la ceniza del año anterior hecha de palma bendita y se unta en la frente en forma de cruz, simbolizando el polvo en el que todos nos hemos de convertir" (Muñoz, 2018, pág. 8). La segunda etapa, inicia el miércoles de tinieblas y culmina el domingo de pascua, es decir la semana mayor, representado todos los momentos de la pasión de Jesucristo.

En un estudio realizado por Moctezuma (2007) se aborda que "la estructura social yaqui y su organización comunitaria gira en torno a las autoridades tradicionales" (p.33), también menciona que "el gobierno civil es la figura predominante del orden interno y en la representación ante los funcionarios del estado mexicano" (p. 34).

La autoridad tradicional de las comuni- 
dades yaquis representan el alto mando en la toma de decisiones, aunque en la mayoría de los casos se realiza un censo para conocer las opiniones del pueblo, cada domingo se realizan asambleas en la guardia tradicional donde se discuten las problemáticas o asuntos internos de la comunidad.

Método

El presente estudio es de alcance descriptivo desde un enfoque cualitativo, ya que el objetivo principal es conocer la perspectiva que tienen los promotores culturales yaquis con relación a necesidades, la función y actividades de los centros de cultura en cada pueblo.

\section{Sujetos}

Para la muestra se entrevistó al 100\% de los promotores culturales de los Centros de Cultura Popular de la comunidad yaqui, en el siguiente orden:

Centro de cultura yaqui Capitán Santiago Valencia Amarillas

Loma de Guamúchil, tradicionalmente Pueblo de Cócorit, Cajeme, Sonora

Promotor: Salustriano Matuz González.

Centro de cultura yaqui Comandante José Bacasegua Seamo

Pueblo de Ráhum

Promotor: José Luis Medina Valencia.

Centro de cultura yaqui Capitán Manuel León Molina

Pueblo de Tórim

Promotora: María del Rosario Hernández Urzúa.

Centro de Cultura Yaqui General Ignacio Mori Seamo

Pueblo de Pithaya

Promotora: Reyna Valencia González.

Centro de cultura yaqui Primer Gobernador Jesús Quintero Valencia

Pueblo de Huírivis

Promotora: Severa Mátuz Valdéz.
Centro de cultura yaqui "Capitán Juan María Santemea"

Comunidad de Pótam Rio Yaqui, Guaymas, Sonora

Jubilado: Ismael Castillo Rendón.

Todos los promotores cuentan con más de 30 años de servicio como empleados adscritos a la Dirección General de Culturas Populares, Indígenas y Urbanas y se encargan de programar, coordinar e impartir los talleres y eventos. También se entrevistó a la directora del Centros de Culturas Populares e Indígenas de Cajeme, quien es fundadora de los centro de cultura de la comunidad yaqui.

Materiales

Se realizó una entrevista semiestructurada compuesto por 19 preguntas divididas en cuatro categorías: Función del centro de cultura, enseñanza aprendizaje, financiamiento y estrategias para la salvaguardia del patrimonio cultural.

Procedimiento

El trabajo de campo se llevó a cabo en el mes de junio del año 2019, en las instalaciones de cada centro de cultura, en donde además de entrevistar a los promotores, se presenció la inauguración de exposiciones diversas organizadas en cada pueblo: Tórim, teopo Jiak joora e etejori into matuj jiyokiam (Lugares ceremoniales y leyendas pintadas al carbón); Pótam, jume juyam intok animaalim yee jittome (plantas y animales que curan); Rahúm, jume juyam intok animaalim yee jittome (plantas y animales que curan); Hurivis, itom jiak yeuri (juguete tradicional yaqui); Pithaya, u baaka itom yoo luturiapo (el uso del carrizo en la tradición). La recolección de información fue de corte transversal.

Resultados

Se detectó que los Centros de Cultura Popular Yaqui son los únicos espacios que congregan a los habitantes de la comunidad yaqui para realizar actividades cultu- 
rales y artísticas de manera permanente; se ofrecen talleres, sin costo, de lunes a sábado, regularmente después de las 15 horas y están dirigidos, en su mayoría, a los niños y jóvenes, porque, en palabras de los promotores, de "ésta manera aprenden a valorar las tradiciones y costumbres"; por lo que se ofertan talleres como lengua materna, música tradicional (violín, guitarra popular), artesanía, elaboración de cabezas de venado con papel, dibujo, costura, elaboración de muñecas, danza tradicional, entre otras manifestaciones de la cultura material e inmaterial. También se observó que en algunos centros, resguardan instrumentos tradicionales (arpas, guitarras, violines, sonajas, tambores), mapas antiguos y fotografías; entre sus actividades cotidianas, presentan de forma temporal exposiciones de los productos elaborados en los talleres, también sobre las tradiciones y costumbres. Cuentan con acervo bibliográfico básico (de historia, literatura, filosofía, productos de investigaciones de la etnia obsequiados por los mismos investigadores) el cual ha sido donado (en su mayoría) o proporcionado por el Instituto Sonorense de Cultura.

En sus espacios, tanto interiores como en exterior, se realizan encuentros coordinados por el Centro de Culturas Populares e Indígenas de Cajeme. Uno de los más importantes, según palabras de la Directora de Culturas Populares de Cajeme, es el Encuentro de Venado y Pascola, porque de esta manera los músicos y danzantes yaquis se organizan para realizar las representación y generar lazos entre los habitantes de los diferentes pueblos, ya que a pesar de que se encuentran a pocos kilómetros de distancia, no se conocen del todo. También se lleva a cabo otro evento de suma importancia para la protección de la salvaguardia del patrimonio cultural inmaterial, el Encuentro de Música Popular Yaqui, en el que se dan cita los diferentes músicos, tanto tradicionales como populares, en el centro cultural que haya sido elegido previamente como sede. El
Encuentro se realiza desde 1987, con el propósito de promover el talento y la creación musical y literaria en su idioma (cahíta) y contribuir a la conservación de una identidad cultural.

Con relación a las categorías estudiadas, los promotores culturales mencionaron con mayor frecuencia que la función del centro cultural es rescatar las costumbres, fortalecer la cultura y las tradiciones; los talleres y actividades de capacitación están dirigidos mayormente a los niños y jóvenes porque en ellos está el compromiso de continuar las prácticas culturales que caracterizan a la comunidad yaqui.

En el rubro enseñanza-aprendizaje, se mencionó que para difundir los talleres se utilizan tres formas de comunicación: 1) Invitación personal, 2) Pegar un cartel escrito a mano, con las particulares del taller que se ofrece y 3) Presentar a las autoridades las actividades programadas en el centro de cultura. También se abordó que se toma en cuenta los intereses particulares de los niños para desarrollar actividades. La metodología de enseñanza empleada por los promotores, quienes también fungen como docentes, es la imitación: comparten lo que saben y cómo les fue enseñado. Los talleres se realizan con el fin de concientizar acerca de la preservación del patrimonio cultural inmaterial con mayor acentuación en la lengua. Con relación al financiamiento, se mencionó que la compra de materiales (hojas, pizarrón, pinturas, entre otros consumibles) y desarrollar proyectos culturales lo adquieren a través del Programa de Apoyo a las Culturas Municipales y Comunitarias (PACMyC) y de aportación propia. No cuentan con donaciones continuas, aunque sí lo han tenido de manera esporádica, y tampoco con otro fondo económico permanente para los gastos de operación del centro.

Por último, en estrategias para la salvaguardia del patrimonio cultural, se encontró que los eventos considerados por los promotores culturales como más importantes son: El Encuentro de Pascola y 
Venado y el Encuentro de Música Popular Yaqui, ambos coordinados por el Centro de Culturas Populares e Indígenas de Cajeme, porque a través de las actividades programadas se revitaliza, difunde y se generan acuerdos para la preservación del patrimonio cultural entre los mismos habitantes de los ochos pueblos yaquis. Estos encuentros se realizan una vez al año y se cuenta, además, con la visita de investigadores, fotógrafos, documentalistas y público proveniente de las zonas aledañas. Se establecen diálogos en la lengua donde se enfatiza la importancia de conservar las tradiciones con el objetivo de "mantener en alto nuestra identidad como indígenas yaquis, eso es lo fundamental, proteger nuestra identidad" [sic] (Promotor cultural de Ráhum).

\section{Conclusiones}

Los Centros de Cultura Popular yaqui desarrollan una función vital para la preservación del patrimonio cultural, porque, como se mencionó en párrafos anteriores, son la única opción de formación artística y de sensibilización hacia las tradiciones y valores de la comunidad existente en cada pueblo. El compromiso de los pro- motores es muy valioso ya que a pesar de las condiciones económicas que son canalizados a los consumibles básicos, su compromiso es grande al tener siempre las puertas abiertas para realizar actividades culturales y que las nuevas generaciones se acerquen a los centros. Dentro de los logros importantes, se puede mencionar el proyecto musical Jiak Usim Jiawai (Armonía de Jóvenes Yaquis) que es un mariachi juvenil-feminil formado en el centro cultural del poblado de Pótam. Las jóvenes fueron capacitadas en el manejo de las cuerdas (violín, vihuela, guitarrón y guitarra) por el promotor cultural y músico tradicional, Ismael Castillo Rendón, y que actualmente continúa en la dirección del proyecto teniendo diversas participaciones en fiestas tradicionales, festivales y eventos particulares interpretando música en la lengua y popular mexicana. A manera de conclusión, se considera muy importante generar mayores lazos entre los gestores culturales externos para desarrollar proyectos de capacitación, actualización para que continúen activos como hasta hoy en día lo han hecho desde 1983.

\section{Referencias}

Castro, T. (2016). Breve diagnóstico de la Unidad Regional Sonora de Culturas

Populares. Secretaría de Educación, Dirección General de Culturas Populares, Unidad

Regional Sonora. Sonora, México. Editorial: ISC.

Castro, T. (2010). Etnias de Sonora. Sonora, México. Editorial: ISC.

De Carli, G. (2012). ¿Museo, centro cultural o ambos?. Recuperado de:

http://www.lacult.unesco.org/docc/museo_centro_cultural_o_ambos.pdf

ISC (s.f.). Centros de Culturas Populares e Indígenas de Cajeme. Recuperado de:

http://isc.gob.mx/devel/patrimonio-sonora/centros-de-cultura-yaqui/

ISC (s.f.). Dirección de Culturas Populares. Recuperado de:

http://isc.gob.mx/devel/patrimoniosonora/direccion-general-culturas- populares/

INPI (2018). Etnografía del pueblo yaqui de Sonora. Recuperado de:

https://www.gob.mx/inpi/es/articulos/etnografia-del-pueblo-yaqui-de-

Moctezuma, J. (2007). Yaquis: Pueblos indígenas del México contemporáneo.

México: Comisión Nacional para el Desarrollo de los Pueblos Indígenas.

Muñoz, M. (2018). Ceremonias y Fiestas Tradicionales: Pueblos Indígenas de

Sonora. Sonora, México: Editorial Lutisuc

Vives, P. (2009). Glosario Crítico de Gestión Cultural. Granada, Comares 